\title{
ANALYSIS OF NORMAL AND SKEW QUADRUPOLE ERRORS AT THE ESRF
}

\author{
R. Nagaoka, L. Farvacque, ESRF, Grenoble, France
}

\begin{abstract}
The paper summarises the methods taken and analysis made so far on the normal and skew quadrupole errors at the ESRF. With the conventional response matrix approach, normal and skew quadrupole errors are extracted respectively from the diagonal and off-diagonal parts of the matrix. Independently, the thousand-turn BPM diagnostic implemented at the ESRF is used to measure the optical asymmetry and the betatron coupling. The excellent accuracy of the turn-by-turn BPMs allows the coupling to be measured by constructing the phase space and performing the normal mode decomposition. The consistency of the results from the two methods is examined. An attempt is made to trace the origin of the obtained errors.
\end{abstract}

\section{INTRODUCTION}

To fully exploit the designed performance of the storage ring, the correction of the optics asymmetry arising from normal quadrupole errors, as well as of the betatron coupling due to skew quadrupole errors is of great importance. At the ESRF, in which there are 96 steerers in each plane and 224 BPMs, the orbit response matrix was initially utilised in this context to calibrate the main quadrupoles up to $10^{-4}$ precision [1]. Averages of the diagonal elements were taken over superperiods and steerers were calibrated at the same time. By utilising the off-diagonal elements (either horizontal to vertical or vice versa), skew quadrupole errors were then deduced to describe and correct the coupling [2]. Upon its success, the diagonal parts have been re-investigated to extract normal quadrupole errors, on the basis of the previously obtained averaged model.

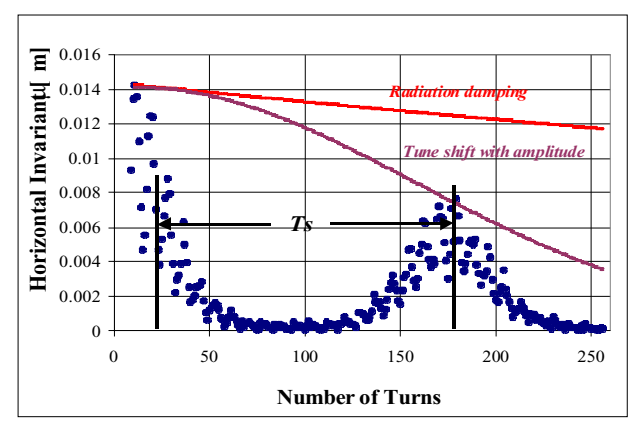

Figure 1: Decoherence of the centre of mass signal measured with the turn-by-turn BPMs.

A diagnostic tool called Mille-Tour BPMs (MTBPMs) was implemented at the ESRF, which reconstructs a turn- by-turn trajectory from readings of the individual pickup electrodes [3]. Although application is limited to reproducible motions, averaging along with an excellent reading accuracy of the ESRF BPMs achieves a highly accurate reading on the turn-by-turn basis. The measurement is triggered with a kicker excitation. In the real application, the measurement is perturbed by the decoherence of the centre of mass signal (Fig. 1), which comes from tune shifts with amplitude, with momentum (chromatic modulation), as well as from the head-tail damping. To minimise them, measurements are made normally with a low beam current in $1 / 3$ filling, a small oscillation amplitude given by a horizontal injection kicker, and a sextupole setting that gives zero chromaticities and minimal tune shift with amplitude.

The MTBPM data provides complementary analysis of the optics asymmetry and the coupling. In the following two sections, the results using these two different methods are reported, respectively on the asymmetry and the coupling. A summary is given in Sec. 4 .

\section{NORMAL QUADRUPOLE ERRORS}

Quadrupole errors are deduced by fitting the diagonal response matrix of the model to the measured (as done in Ref. 4). The fit is started from the symmetrical solution and the steerer calibration found in the averaged response matrix analysis. An iterative solution of the linearised equation is made with the SVD method. At each step, the optics is updated with the obtained quadrupole increments, and the number of eigenvectors used in the inversion is appropriately increased. The process is continued until convergence (saturation).
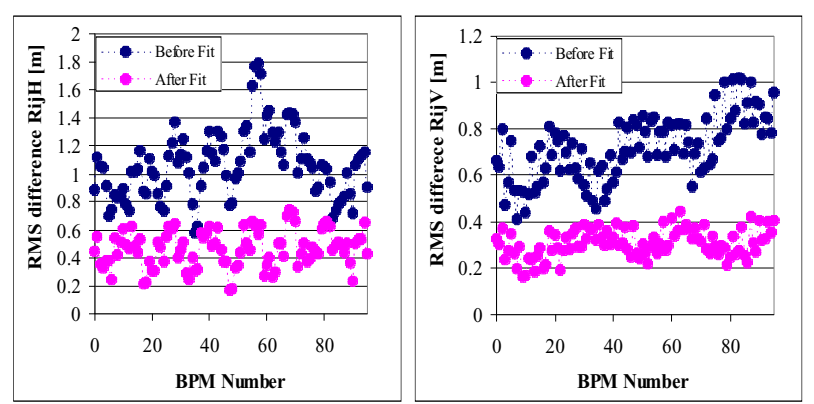

Figure 2: Difference in the response matrix between the model and the measured. For a given BPM, rms is taken over all steerers. Left: horizontal. Right: vertical.

Application was made to the response matrix measured with all quadrupole correctors switched off. The solution found gives a reasonably good fit in both planes (Figs. 2). Computed beta asymmetry (horizontal) is in good 
agreement with that obtained with the turn-by-turn BPM analysis (Figs. 3). On the basis of the obtained solution, an online correction of the optical asymmetry is underway. To be able to iterate the correction, a partial response matrix acquisition on a limited number of steerers is to be made to speed up the process. The link of this scheme to the conventional correction of half-integer resonances shall be pursued.
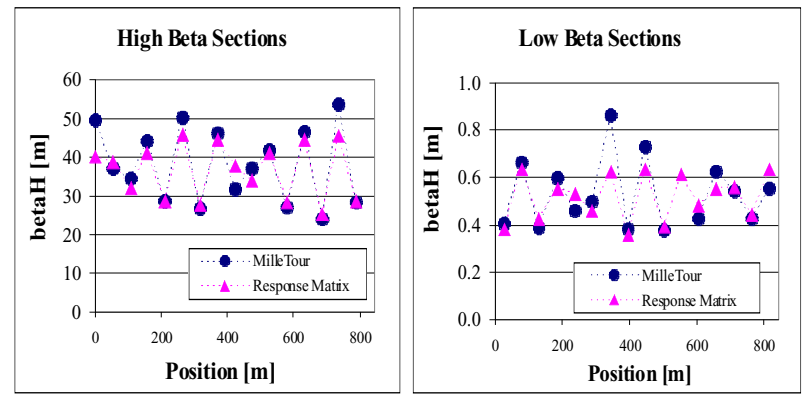

Figure 3: Beta asymmetry deduced with the response matrix and MTBPMs. All quad correctors are turned off.

From the turn-by-turn BPM data, Twiss parameters and the invariant of motion are extracted by constructing the phase space at every straight section in which there are two BPMs on both ends. The betatron phase advance is obtained by computing for each BPM, the spectral amplitude and phase of the motion on the tune frequency.

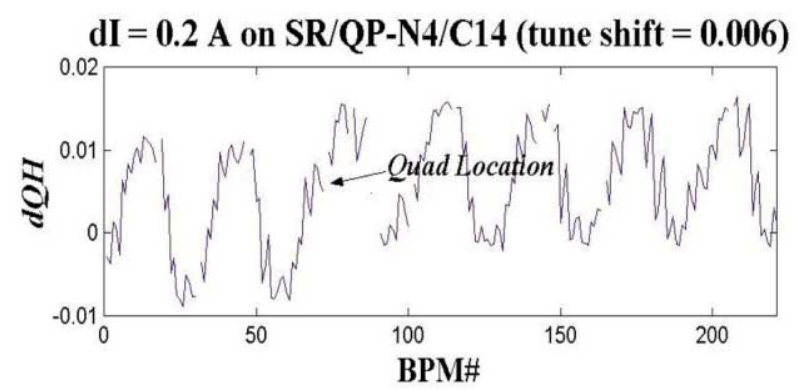

Figure 4: Detection of a quad error in the ring, through betatron phase advance measured with MTBPMs.

The sensitivity of MTBPMs in the betatron phase measurement was explored. By changing the strength of a quadrupole corrector by the equivalent of a gradient error of $2 \times 10^{-3}$ in the strongest quadrupole, the difference in the phase advance with and without the error was analysed. On top of the beating of the phase difference by 7 periods, which comes from the combination of the optics and the machine structure, the result showed a discrete jump at the location of the error (Fig. 4), demonstrating the capability of the quadrupole error detection of this order. On the basis of this finding, the origin of the observed betatron tune shift with the beam current was investigated, by taking the difference in phase advance between 5 and $200 \mathrm{~mA}$. As opposed to the former, the result showed a smooth increase in the phase difference (Fig. 5), which is consistent with the hypothesis that the tune shift is due to the wake field induced by the low-gap vacuum chambers, which are distributed around the ring [5].

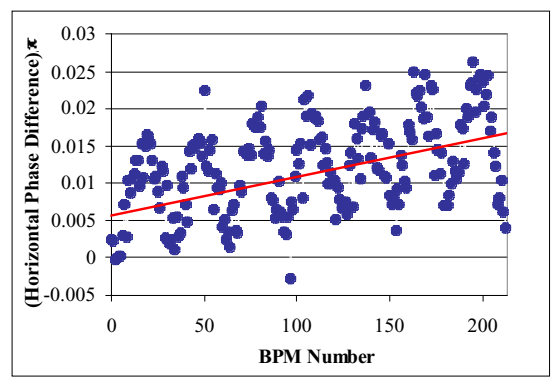

Figure 5: Difference of betatron phase advance between 5 and $200 \mathrm{~mA}$ measured with MTBPMs.

\section{SKEW QUADRUPOLE ERRORS}

In the transversely coupled equations of orbit response to a steerer, skew quadrupoles appear linearly in the plane orthogonal to the steerer excitation, and therefore can be solved via the standard matrix inversion. Carrying it out in the specific ESRF case, despite not resolving a single magnet skew error, locally integrated strengths, namely the effective skew error distribution, can be obtained reliably, with which the coupling of the machine can be described locally and globally in a satisfactory manner. The correction of coupling has successfully been made using the obtained distribution [2].

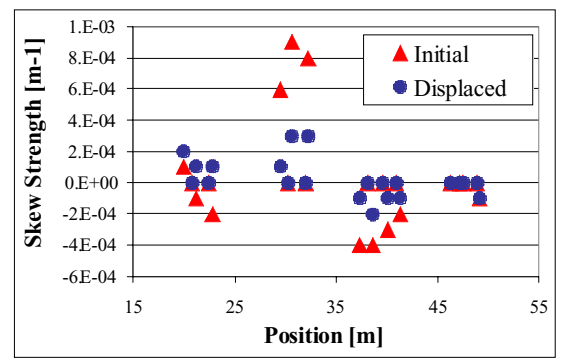

Figure 6: Measured reduction of peak skew errors.

The obtained skew errors have been found to vary little over years of time despite occasional realignment works. Assuming that they are originating from quadrupole rotation errors, the rms tilt angle is computed to be $\theta_{r m s}=$ $0.35 \mathrm{mrad}$, which indicates that the peaks are too large to be girder rotation errors. An attempt was made to eliminate the two largest peaks in the distribution. Among the two equivalent actions of rotating a quadrupole and vertically displacing a sextupole, the latter was chosen for the reason of feasibility. Displacing three sextupoles by nearly $0.5 \mathrm{~mm}$ in the proper directions, both peaks were successfully removed to a good extent (Fig. 6), which reduced the geometric coupling of the uncorrected machine from 7.5 to $4 \%$, as well as enabling the standard correction to reach a lower vertical emittance. Efforts were made to distinguish the contribution of sextupoles to the skew errors from quadrupoles. By exploring sextupole 
settings with a sufficiently large difference on a single family, keeping feasibility of beam accumulation, the skew error analysis is repeated singling out the chosen sextupole family.

By making full use of the excellent accuracy of MTBPMs, an attempt was made to measure the betatron coupling through the normal mode decomposition [6]. The developed steps are as follows: For each straight section that has no focusing element in between two BPMs, 1) Construct the phase space (x, x', z, z'). 2) Fit the phase space data to extract a $4 \times 4$ one turn matrix. 3 ) Perform the normal mode decomposition to obtain the normal modes as well as the rotation matrix that transforms the geometric modes to the former. The second step was found to be the most non-trivial, especially as the number of available turns is severely limited by the strong decoherence of the beam. Since the matrix can be obtained using only 4 independent turns, the data over the first 20 turns were used to increase the precision through averaging. It was also found important in many cases to impose the symplectic condition to be fulfilled by the one turn matrix, to extract matrix elements that are more ambiguous due to smallness of the vertical amplitudes.
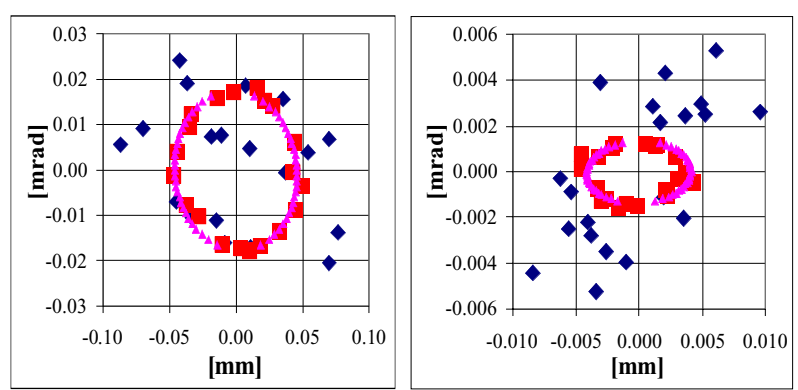

Figure 7: Two examples showing the transformation from the vertical phase space (blue) to the normal mode (red), and the fit of the normal mode (pink triangles). Measured vertical emittances are 30 (left) and $8 \mathrm{pm} \cdot \operatorname{rad}$ (right).

The correctness of the results can be readily seen in the decoupling of the two normal modes (Figs. 7), as well as in constancy of the ratio of the two normal mode ellipses around the machine. The application was successful over the entire range of coupling, surprisingly down to the lowest coupling, where the vertical emittance of $8 \mathrm{pm} \cdot \mathrm{rad}(0.2 \%$ emittance coupling) was measured with a pinhole camera. The decomposition at the ultimately low coupling requires perfect machine stability and fails as soon as the beam is perturbed by external noises.

Although the ratio of the two normal mode ellipses in most cases closely followed the measured emittance coupling, it strictly depends on the initial condition. The magnitude of off-diagonal elements in the rotation matrix, instead, represents the local coupling of the machine. The present scheme thus opened a new possibility of the coupling correction with two advantages, one that the coupling can be measured at all ranges, and the other that it enables a global correction. The response matrix approach, despite offering an accurate model of the coupling, could only measure the initial large coupling, while smaller couplings had to be measured with pinholes, which are located at only two positions in the ring. The first tests were successful, managing corrections in both high and low coupling regimes.

Analysis of normal and skew quadrupole errors was attempted with MTBPMs. By introducing the flags in between BPMs, their strengths were fitted to reproduce the remaining data with respect to the phase space at straight sections. On the simulation, this method was found to reproduce well the known error distribution. Applying it to the real data, however, it turned out that small unknown calibration differences in the BPMs critically spoil the fit. A scheme is being developed to deduce firstly the plausible calibration coefficients of the BPMs by imposing that quantities such as the betatron phase advance computed from local displacements match those obtained by other means (Fourier analysis).

\section{CONCLUSION}

Normal and skew quadrupole errors have been deduced from the diagonal and off-diagonal parts of the orbit response matrix, respectively. The optical asymmetry and the linear coupling described by the resultant error distributions are both in good agreement with those obtained with the MTBPM data. Thanks to the extraordinary precision provided by the MTBPMs, the normal mode decomposition to analyse the coupling could be made down to the limiting coupling of $8 \mathrm{pm} \cdot \mathrm{rad}$ vertical emittance. A scheme is being developed to deduce normal and skew quadrupole errors from the turnby-turn BPM data.

\section{ACKNOWLEDGEMENT}

The authors thank Kees Scheidt, the founder of MTBPMs, for his active participation to the measurement and analysis using the developed tool.

\section{REFERENCES}

[1] L. Farvacque, R. Nagaoka, "Calibration of Quadrupole Magnets via Response Matrix Fitting”, EPAC98, Stockholm, June 1998.

[2] R. Nagaoka, "Work Carried Out at the ESRF to Characterise and Correct the Coupling", EPAC paper, Vienna, 2000.

[3] L. Farvacque, R. Nagaoka, K. Scheidt, "Breaking New Ground with High Resolution Turn-by-Turn BPMs at the ESRF", DIPAC 2001, ESRF, Grenoble.

[4] J. Safranek, "Experimental Determination of Storage Ring Optics Using Orbit Response Measurements", Nucl. Instr. And Meth., A388 (1997) 27.

[5] R. Nagaoka, "Impact of resistive-wall wake fields generated by low-gap chambers on the beam at the ESRF", this conference.

[6] D. Edwards and L. Teng, "Parameterization of Linear Coupled Motion in Periodic Systems", IEEE Transactions on Nuclear Science, NS-20, No. 3, June 1973. 